\title{
GLoBES
}

\section{General Long Baseline Experiment Simulator}

\author{
Patrick Huber \\ Department of Physics, Virginia Tech, Blacksburg, VA 24062, USA \\ E-mail: pahuberevt.edu \\ Joachim Kopp* \\ Max-Planck-Institut für Kernphysik, Postfach 1039 80, 69029 Heidelberg, Germany \\ E-mail: jkopp@mpi-hd.mpg.de
}

\section{Manfred Lindner}

Max-Planck-Institut für Kernphysik, Postfach 1039 80, 69029 Heidelberg, Germany

E-mail: lindner@mpi-hd.mpg.de

\section{Walter Winter ${ }^{\dagger}$}

Institut für theoretische Physik und Astrophysik, Universität Würzburg, 97074 Würzburg, Am Hubland, Germany

E-mail: winter@physik.uni-wuerzburg.de

We present the basic components of GLoBES ("General Long Baseline Experiment Simulator"), which is a multi-purpose software for the simulation of future long baseline and reactor experiments. We illustrate the building blocks of the Abstract Experiment Definition Language (AEDL), and we describe the main features of the $\mathrm{C}$ user interface. In addition, we discuss recent developments in GLoBES, such as major new features in GLoBES 3.0.

10th International Workshop on Neutrino Factories, Super beams and Beta beams June 30 - July 52008

Valencia, Spain

${ }^{*}$ Poster artwork and design

† Speaker. 

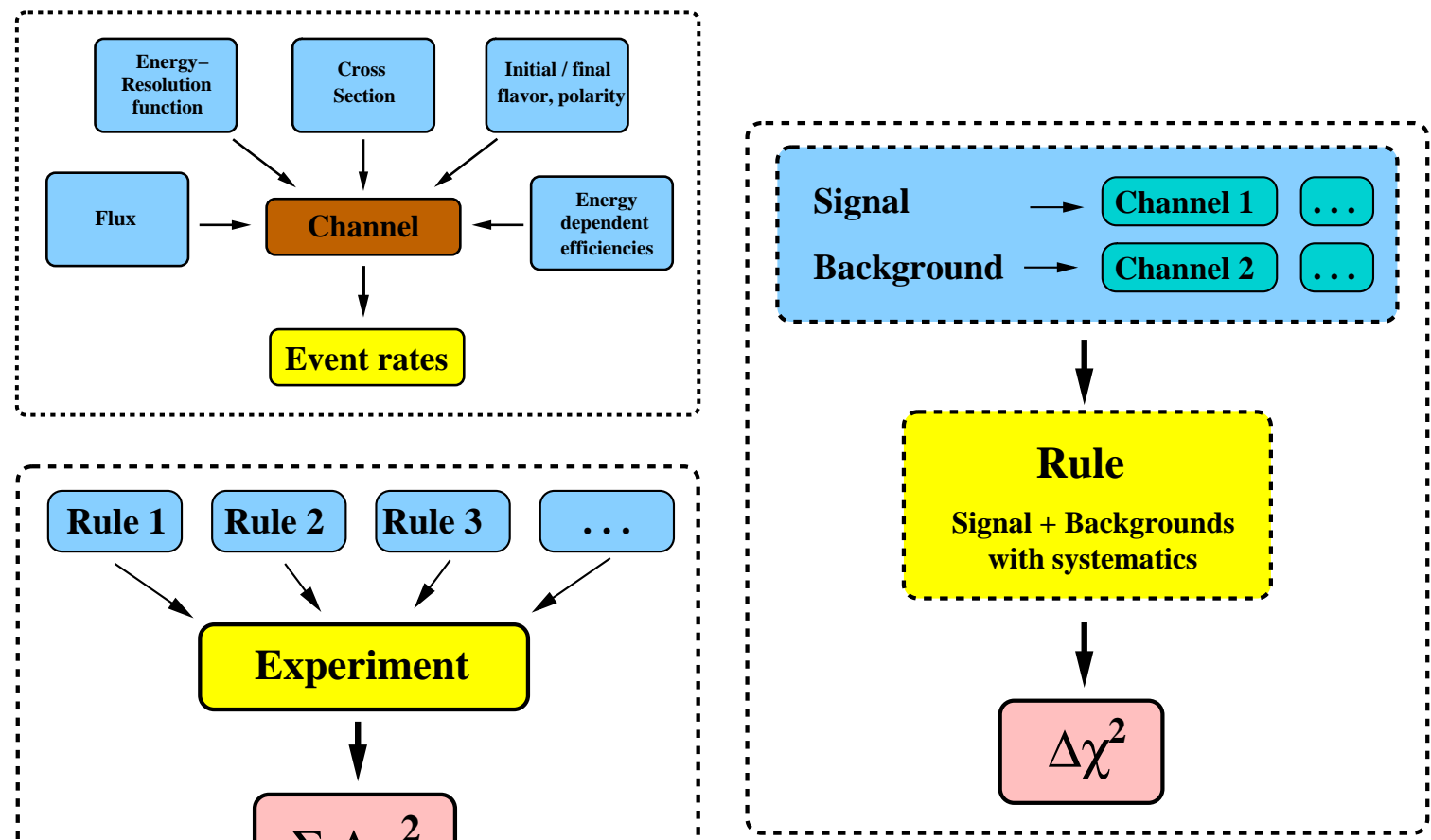

Figure 1: Principle building blocks for experiment definition in GLoBES: Channel (upper left), rule (right), and experiment (lower left). Figures from Ref. [1].

GLoBES [1,2] is a multi-purpose software for the simulation of individual long-baseline and reactor neutrino oscillation experiments, as well as for the global analysis of multiple experiments. It includes the treatment of statistics, systematics, correlations, and degeneracies (see Ref. [3] for a classification). It consists of two major components: Abstract Experiment Definition Language (AEDL) describes individual experiments using plain text files, and a user interface (C library) for the $\chi^{2}$ analysis, which loads one or more AEDL files and provides the functionality for the statistical analysis. The separation between AEDL and the user interface makes GLoBES an interesting tool for both the experimentalist and theorist. For example, the theorist may use pre-defined files for the simulation of new, potentially interesting physics effects. The experimentalist, on the other hand, can quickly test the effect of small modification in the experiment definition on physics. Note that GLoBES is not meant to replace a full Monte Carlo simulation of the experiment, but has to be understood as a tool to identify the key parameters and critical factors for especially future experiments. For example, the detector is simulated by an effective response function, which describes its behavior on a statistical basis. This response function can be used from Monte Carlo simulations as an input for GLoBES.

AEDL describes an experiment, such as by source type and spectrum, matter density profile, cross sections, detector properties (efficiencies, energy resolution, backgrounds), and systematics. It uses three three building blocks, as illustrated in Fig. 1: A channel links a produced flavor state with a certain flux, via the oscillation physics, to the detection with a specific interaction type. It 
results in the event rate of this interaction type. A rule combines the event rates from different channels, which can either be signal or background for that rule, with a specific systematics; it results in a $\Delta \chi^{2}$. An experiment contains one or more rules, which are combined to the total $\Delta \chi^{2}$. It shares certain characteristics among the rules, such as baseline and matter density profile, but not the systematical errors. For example, a simple neutrino factory may store $\mu^{+}$, which leads to $v_{e}$ and $\bar{v}_{\mu}$ in the beam. A signal channel might be $v_{e} \rightarrow v_{\mu}$, which can be combined into an appearance rule with the background channel $\bar{v}_{\mu} \rightarrow \bar{v}_{\mu}$ (for the charge mis-identified events) leading to a $\Delta \chi^{2}$. An experiment may contain more such rules, such as for different appearance and disappearance channels and different polarities.

One or more descriptions of experiments can be loaded by the $\mathrm{C}$ user interface. This interface provides the functionality to extract physical information from the simulated event rate spectra. For example, it allows for sections and projections (marginalizations) of the multi-dimensional fit manifold, i.e., it allows for the inclusion of correlations and degeneracies. Of course, one can also obtain low-level information, such as oscillation probabilities and event rates. New features in GLoBES 3.0 are the fully customizable systematics interface supporting multiple sources and detectors (such as for reactor experiments), and the fully customizable external input to be added to the $\chi^{2}$ before marginalization. Heart of the $\chi^{2}$ analysis is the oscillation and rate engine, which includes a full three flavor treatment, the use of arbitrary matter density profiles, and an extremely high numerical efficiency with specifically designed numerical algorithms, such as Ref. [4]. The oscillation engine can be modified as well, which allows for the simulation of new physics effects.

The results obtained with the GLoBES software can nowadays be found in the core of many long baseline experiment studies and major collaborative efforts, such as the international neutrino factory and superbeam scoping study (ISS) [5] and the US long baseline neutrino experiment study [6].

In summary, the GLoBES software is a multi-purpose tool for the simulation of future long baseline and reactor neutrino experiments, which has been especially designed for the comparison and combination of different options on equal footing. The software has been established in the community, which, for example, means that results obtained with GLoBES can now be found in many strategy papers. With the version 3.0, it has reached a new level of flexibility at all levels. The further development of GLoBES will depend on user demands and the requirements of the neutrino oscillation community.

\section{References}

[1] P. Huber, M. Lindner, and W. Winter, Comput. Phys. Commun. 167, 195 (2005), hep-ph / 0407333.

[2] P. Huber, J. Kopp, M. Lindner, M. Rolinec, and W. Winter, Comput. Phys. Commun. 177, 432 (2007), hep-ph/0701187.

[3] P. Huber, M. Lindner, and W. Winter, Nucl. Phys. B645, 3 (2002), hep-ph / 0204352.

[4] J. Kopp, Int. J. Mod. Phys. C19, 523 (2008), physics / 0610206.

[5] A. Bandyopadhyay et al. (ISS Physics Working Group), arXiv:0710.4947 [hep-ph].

[6] V. Barger et al., arXiv:0705.4396 [hep-ph]. 